\title{
Wo bleiben die Kinder, wenn der ausländische Elternteil in seine Heimat zurückkehren will?
}

\author{
Dr. Magdalena Dollinger \\ Mitglied der Kommission Zivil-, Familien- und Erbrecht, Recht an- \\ derer Lebensgemeinschaften des djb; Rechtsanwältin, München
}

Die attraktive Dottoressa und der charmante Archäologe lernten sich auf einer Expedition in Äthiopien kennen. Seinetwegen zog sie aus ihrer Heimatstadt Mailand nach München, startete hier ihre Karriere als Managerin, man lebte in gehobenen Verhältnissen, und das Glück war perfekt, als zwei Söhne geboren wurden. Doch es währte nur kurz. Nach erfolgloser Eheberatung ${ }^{1}$ zog der Gatte schließlich aus; wegen des Umgangs eskalierte der Streit, bis die Mutter das Aufenthaltsbestimmungsrecht beantragte, um mit den Kindern nach Italien zu ziehen. Als ihr das versagt wurde, ${ }^{2}$ verschwand sie des Nachts vor Schulbeginn mit den Kindern. Nach einem halben Jahr wurden die Kinder nach HKÜ-Verfahren zum Vater zurückgebracht und sie besuchten in Deutschland wieder ihre alten Schulen. Zehn Monate später passte die Mutter die Knaben ab, zerrte sie ins Auto und versteckte sie so, dass die Polizei sie nicht mehr finden konnte. Erst als sie in Haft genommen wurde, verriet ihr Bruder, dass sich die Kinder mit ihrer Oma in Slowenien aufhielten. Neun Monate waren sie verschwunden und wurden nicht unterrichtet. Derzeit leben sie wieder bei ihrem Vater.

Dieser Fall ist nur deshalb so dramatisch verlaufen, weil die Mutter mit den Kindern ins Ausland verziehen wollte. Denn das OLG hätte ihr das Aufenthaltsbestimmungsrecht übertragen, wenn nicht hätte befürchtet werden müssen, dass der Vater sein Umgangsrecht, das schon hierzulande nur dank eines energischen Umgangspflegers einigermaßen ausgeübt werden konnte, in Italien nicht gänzlich verlöre. ${ }^{3}$ Ein Umzug ans andere Ende der Republik wäre also kein Auslöser für das Unglück der Kinder geworden.

Will ein Elternteil mit seinem Kind ausreisen, so ist das kein rechtliches Problem, wenn er die alleinige elterliche Sorge hat. $^{4}$

Das Umgangsrecht $\mathbb{} 1626$ Absatz 3 BGB steht dem regelmäßig nicht entgegen. ${ }^{5}$ Der umgangsberechtigte andere Elternteil hat Einschränkungen hinzunehmen und wird darauf verwiesen, dass er die Kontakte mit dem Kind auch auf Entfernung ausüben kann, gegebenenfalls in anderer Ausgestaltung, er z.B. verlängerte Ferienaufenthalte wahrnimmt anstatt regelmäßiger Wochenendkontakte.

Besteht gemeinsame elterliche Sorge, $\mathbb{S} 1626$ BGB, und stimmt der mitsorgeberechtigte Elternteil dem Wegzug des Kindes nicht zu, so muss die oder der Ausreisewillige das Aufenthaltsbestimmungsrecht gemäß $\$ 1671$ BGB auf sich allein übertragen lassen, denn der Entzug eines Kindes bei gemeinsamer Sorge ohne Einverständnis des anderen Elternteils stellt eine Kindesentführung im Sinne des $\mathbb{2} 35$ StGB dar.
Es geht also in diesen Streitfällen um das alleinige Aufenthaltsbestimmungsrecht. In der Vielzahl der Fälle, selbst bei Auswanderungen in ferne Länder, wurde erstaunlicherweise selten das gesamte Sorgerecht beantragt. Der Grund dürfte darin liegen, dass es eines Gesamtentzuges des Sorgerechts nicht bedarf und ein Teilbereich leichter zu erlangen ist als die gesamte elterliche Sorge, denn Teilregelungen sind das mildere Mittel im Sinne der Verhältnismäßigkeit. ${ }^{6}$ Schon kraft des Aufenthaltsbestimmungsrechtes entsteht nach herrschender Meinung die Berechtigung, über den Aufenthalt des Kindes allein zu entscheiden. ${ }^{7}$ Nur unter besonderen Umständen kann dann das Verbringen des Kindes ins Ausland durch den Elternteil, der das Aufenthaltsbestimmungsrecht inne hat, rechtswidrig sein. ${ }^{8}$ Die Entscheidung richtet sich nach $\ 1671$ Absatz $2 \mathrm{Nr}$. 2 BGB, wonach der Antragstellerin oder dem Antragsteller das Aufenthaltsbestimmungsrecht zu übertragen ist, wenn zu erwarten ist, dass die Aufhebung der gemeinsamen Sorge und die Übertragung auf einen Elternteil dem Wohle des Kindes am besten entspricht. Kriterium ist also das Kindeswohl - wie in allen Bereichen der elterlichen Sorge. ${ }^{9}$

Nun gilt aber in Fällen binationaler Eltern für den ausreisewilligen Teil auch sein Recht auf Freizügigkeit, Ausfluss der allgemeinen Handlungsfreiheit aus Artikel 2 GG, welches ihm gestattet, grundsätzlich jederzeit über seinen Aufenthalt zu bestimmen und in sein Heimatland zurückzukehren. In Rechtsprechung und Literatur war lange umstritten, wie - auch unter Berücksichtigung des vorrangigen Kindeswohles - dieses Recht des ausländischen Elternteiles zu gewichten sei. Das OLG Zweibrücken ${ }^{10}$ und das OLG Köln ${ }^{11}$ hatten entschie-

1 Zu den Problemen, denen ausländische Partnerinnen oder Partner ausgesetzt sind, wenn sie ihrem deutschen Mann oder ihrer deutschen Frau hierher folgen, vgl. Curvello, Tatiana Lima, Psychologische Beratung mit bikulturellen Paaren und Familien, in: iaf e.V., Die Balance finden, Frankfurt a.M., 2008. Sie bezeichnet es als eine große Aufgabe, sich hier ein gemeinsames Leben aufzubauen.

2 OLG München, FamRZ 2009, S. 794.

3 Zu der Problematik, im Ausland den Umgang durchzusetzen, vgl. Völker, Mallory, FamRZ 2010, S. 1067; S. 157 ff. m.w.N.

$4 \S 1626 a$ Abs. 2 BGB oder $\$ 1671 \mathrm{ff}$. BGB.

5 BGH, NJW-RR 1990, S. $258 \mathrm{ff}$.

6 Vgl. Zuck, Rüdiger, FamRZ 2010, S. 1950.

7 A. A. Staudinger/Rauscher, BGB, 2006, \$1684 Rz. $67 \mathrm{f}$.

8 Vgl. BGH, FamRZ 1999, S. 651. Das OLG Köln, FamRZ 2010, S. 913 hat z.B. einer Inhaberin des Aufenthaltsbestimmungsrechts, einer Thailänderin, untersagt, das Kind in ihre Heimat mitzunehmen, da sie kraft des Aufenthaltsbestimmungsrechtes „nicht schrankenlos über den Aufenthalt des Kindes alleine entscheiden“ dürfe. Die dauerhafte Verlegung des Wohnortes des Kindes in ein außereuropäisches Land (nicht in einen EU-Staat) sei widerrechtlich im Sinne von Art. 3 HKiEntÜ, weil sie die Mitsorge des Vaters in unzulässiger Weise beeinträchtige.

9 Vgl. Zuck, FamRZ 2010, S. 1946 mit Verweis auf BVerfGE 60, 79, 88 = FamRZ 1982, S. 567; BVerfGE 103, 89, 107 = FamRZ 2001, S. 343; BVerfGE 121, 69, 92 = FamRZ 2008, S. 845 .

10 NJW-RR 1990, S. 258.

11 NJW-RR 2006, S. 1588. 
den, dass das Freiheitsrecht eines Elternteils nur eingeschränkt werden kann, wenn das Kindeswohl dies zwingend erfordert. Andere Entscheidungen forderten beachtenswerte, insbesondere berufliche Gründe, die der ausreisewillige Elternteil darlegen musste. ${ }^{12}$ Das OLG Köln sah die Freizügigkeit pflichtgebunden in Hinblick auf das Kindeswohl, es müssten triftige Gründe für den Wegzug vorliegen. ${ }^{13}$ Nur dann sei es auch gerechtfertigt, das Elternrecht des anderen und sein Umgangsrecht einzuschränken.

Der BGH hat nun diese Fragen eindeutig beantwortet. Mit seinem Beschluss vom 28. April $2010^{14}$ hat er klargestellt, dass das Kindeswohl „vornehmlich“ Maßstab der Entscheidung sei. Die allgemeine Handlungsfreiheit des auswanderungswilligen Elternteils schließe es aus, die Möglichkeit seines Verbleibes im Inland als Alternative in Betracht zu ziehen. Die Gründe und Motive für seinen Auswanderungswunsch seien nur insoweit bedeutsam, als sie sich auf das Kindeswohl auswirken. Das wiederum sei aber gegeben, wenn etwa mit der Auswanderung (vorwiegend oder beiläufig) auch der Zweck verfolgt wird, den Kontakt des Kindes mit dem anderen Elternteil zu vereiteln, denn dann ,steht die Bindungstoleranz des betreuenden Elternteils und somit seine Erziehungseignung in Frage“.

Die Kindeswohl ist umfassend zu prüfen: Der gesamte Senat, nicht nur eine Berichterstatterin oder ein Berichterstatter, hat das Kind anzuhören ${ }^{15}$, eine Verfahrenspflegerin oder ein Verfahrenspfleger ist zu bestellen und bei der Anhörung hinzuzuziehen. ${ }^{16}$ Die Entscheidung fällt dann danach, „ob die Auswanderung mit dem Elternteil oder der Verbleib des Kindes beim weiter im Inland ansässigen Elternteil die für das Kindeswohl bessere Lösung ist ${ }^{17}$. Hierbei sind die Bindungen des Kindes zu klären: Überwiegt diejenige an die auswandernde Hauptbezugsperson oder sind es andere Bindungen, zum Beispiel an die gewohnte Umgebung und das gesamte, ver- traute Umfeld, ferner der vom Kind geäußerte Wille (welcher bei kleinen Kindern mehr als Ausdruck ihrer Bindungen und bei älteren eher als Ausdruck ihrer Persönlichkeit zu werten ist). Falls erforderlich, ist ein Sachverständigengutachten einzuholen. Auch ist die Alternative eines Wechsels zum bleibenden Elternteil in Betracht zu ziehen.

Fälle, die brisant sind, wie der eingangs geschilderte, sind zum Glück nicht die Regel. Aber Ehen zwischen deutschen und ausländischen Partnerinnen und Partnern, die in den 50er Jahren des letzten Jahrhunderts noch etwas Exotisches waren, sind heute etwas völlig Normales und recht zahlreich: Im Jahre 2008 waren 10,9 Prozent aller in Deutschland geschlossenen Ehen (41.442 von 377.055) binational, und diese Quote ist konstant. ${ }^{18}$ Von allen in Deutschland geborenen Kindern entstammen zwischen 12 und 13 Prozent binationalen Ehen, das waren beispielsweise im Jahre 200686.744 Kinder. ${ }^{19} \mathrm{Ihr}$ Wohlergehen ist nach den neuen BGH-Entscheidungen das vorrangige und entscheidende Kriterium dafür, ob sie ihren Aufenthalt in Deutschland beenden und mit ihrem ausländischen Elternteil wegziehen (müssen oder dürfen).

12 OLG München, FamRZ 2008, S. 1774, OLG Nürnberg, FamRZ 2010, S. 135.

13 FamRZ 2009, S. 436.

14 FamRZ 2010, S. $1060 \mathrm{ff}$.

15 Dies wurde in einer neueren Entscheidung bestätigt, dass generell der gesamte Senat anhören und dabei die Verfahrenspflegerin oder der Verfahrenspfleger anwesend sein muss, vgl. BGH, FamRZ 2011, S. 800.

16 Wobei sich diese eingehend mit dem Fall befasst haben muss und nicht erst kurz vor dem Termin nur 30 Minuten mit dem Kind gesprochen und die Akten "selektiv“ gelesen haben darf; KG, FamRZ 2011, S. 800.

17 BGH, FamRZ 2010, S. 1063.

18 Auskunft des Bundesministeriums für Familie, Senioren, Frauen und Jugend vom 8.6.2011 an die Autorin.

19 S. iaf, a.a.O, vorletzte Seite.

\section{Erbrecht im Visier: Wann kommt das Erbrecht im 21. Jahrhundert an?}

\section{Renate Maltry}

Vizepräsidentin des djb; Rechtsanwältin, Fachanwältin für Erbrecht, Fachanwältin für Familienrecht, München

Im Vergleich zum Familienrecht, das sich fortlaufend dem gesellschaftlichen Wandel angepasst hat, erstaunt, dass das Erbrecht bisher kaum Veränderung bzw. Anpassung an geänderte gesellschaftliche Strukturen erfahren hat.

Das Erbrecht ist der Teil des BGB, der seit seinem Inkrafttreten im Jahre 1900 mit wenigen Ausnahmen noch unverändert geblieben ist.

Es ist an der Zeit über den Einfluss des familiären und demografischen Wandels im Erbrecht nachzudenken und Refor- men, die einer modernen Gesellschaft gerecht werden, einzufordern.

\section{Demografischer Wandel}

Betrug das durchschnittliche Lebensalter im Jahre 1881 bei Frauen 38,45 Jahre und bei Männern 35,6 Jahre, so hat sich die Lebenserwartung bei Männern um 40 Jahre und bei Frauen um 43 Jahre erhöht. ${ }^{1}$ Folge hiervon ist, dass die sogenannten jungen Alten, also die ca. 60-Jährigen, von den sehr Alten erben.

1 Statistisches Bundesamt: Statistisches Jahrbuch 2007. 